\title{
Fejér and Hermite-Hadamard Type Inequalities for $N$-Quasiconvex Functions
}

\author{
S. Abramovich ${ }^{1 *}$ and L. E. Persson ${ }^{2,3^{* * *}}$ \\ ${ }^{1}$ University of Haifa, Haifa, Israel \\ ${ }^{2}$ Luleå University of Technology, Sweden \\ ${ }^{3}$ UiT. The Arctic University of Norway, Narvik, Norway \\ Received April 10, 2017
}

\begin{abstract}
Some new extensions and refinements of Hermite-Hadamard and Fejér type inequalities for functions which are $N$-quasiconvex are derived and discussed.
\end{abstract}

DOI: $10.1134 / \mathrm{S} 0001434617110013$

Keywords: Fejér inequality, Hermite-Hadamard inequality, $\gamma$-quasiconvexity, convexity.

Dedicated to Professor Stefan Samko on the occasion of his 75 th birthday.

\section{INTRODUCTION}

The Hermite-Hadamard inequality says that for any convex function $f: I \rightarrow \mathbb{R}, I$ an interval and, for $a, b \in I$, one has

$$
f\left(\frac{a+b}{2}\right) \leq \frac{1}{b-a} \int_{a}^{b} f(t) d t \leq \frac{f(a)+f(b)}{2},
$$

and the Fejér inequality reads

$$
f\left(\frac{a+b}{2}\right) \int_{a}^{b} p(x) d x \leq \int_{a}^{b} f(t) p(t) d t \leq \frac{f(a)+f(b)}{2} \int_{a}^{b} p(x) d x
$$

when $f$ is convex and $p:[a, b] \rightarrow \mathbb{R}$ is nonnegative, integrable, and symmetric with respect to the midpoint $x=(a+b) / 2$.

There have been a lot of developments and applications of these inequalities. One such development is to replace the notion of classical convexity by other variants and generalizations of convexity. An early well cited such paper is by Dragomir et. al. [1], also see [2] and [3]. We also mention the paper [4] and especially the book [5] by Niculescu and Persson, where several generalizations, variants and applications are described and placed into a more general convexity context.

In this paper, some new extensions and refinements of Hermite-Hadamard and Fejér type inequalities for functions which are $N$-quasiconvex are provided. We also deal with monotonicity related to Fejér and Hermite-Hadamard type inequalities for 1-quasiconvex functions.

Definition. Let $\gamma$ be a real number. A real-valued function $f$ defined on an interval $[0, b), 0<b \leq \infty$, is said to be $\gamma$-quasiconvex if it can be represented as the product of a convex function and the power function $x^{\gamma}$.

\footnotetext{
*E-mail: abramos@math.haifa.ac.il

${ }^{* *}$ E-mail: Lars-Erik.Persson@ltu.se
} 
In Sec. 2 we extensively use the following results from [6].

Lemma 1. Let $\varphi$ be a convex and differentiable function, and let $\psi_{k}(x)=x^{k} \varphi(x), k=0,1, \ldots, N$. Then the $N$-quasiconvex function $\psi_{N}(x)=x^{N} \varphi(x)$ for $x, y \in[a, b), a \geq 0$, satisfies

$$
\begin{aligned}
\psi_{N}(y)-\psi_{N}(x) & \geq \psi_{N}^{\prime}(x)(y-x)+(y-x)^{2} \sum_{k=1}^{N} y^{k-1} \psi_{N-k}^{\prime}(x) \\
& =\psi_{N}^{\prime}(x)(y-x)+(y-x)^{2} \frac{\partial}{\partial x}\left(\frac{x^{N}-y^{N}}{x-y} \varphi(x)\right) .
\end{aligned}
$$

For $N=1$, for $x \in[a, b)$, we have

$$
\psi_{1}(x)-\psi_{1}(\bar{x}) \geq \psi_{1}^{\prime}(\bar{x})(x-\bar{x})+\varphi^{\prime}(\bar{x})(x-\bar{x})^{2},
$$

from which we obtain the following assertion (also see [7] and Lemma $C$ in [6]) for 1-quasiconvex function $\psi_{1}$ and for integrable nonnegative $f$ satisfying $\int_{a}^{b} f(x) d x>0$.

Theorem A. Let $f$ be a nonnegative function, and let $\varphi$ be a differentiable convex function on $[0, b)$, $0<b \leq \infty$. Let $f$ and $\varphi \circ f$ be $\mu$-integrable functions on the probability measure space $(\Omega, \mu)$ and $\int_{\Omega} f(s) d \mu(s)>0$. Let $\psi_{1}(x)=x \varphi(x)$. Then

$$
\begin{aligned}
\int_{\Omega} \psi_{1}(f(s)) d \mu(s)-\psi_{1}\left(\int_{\Omega} f(s) d \mu(s)\right) \\
\quad \geq \int_{\Omega} \varphi^{\prime}\left(\int_{\Omega} f(\sigma) d \mu(\sigma)\right)\left(f(s)-\int_{\Omega} f(\sigma) d \mu(\sigma)\right)^{2} d \mu(s)
\end{aligned}
$$

holds. If $\varphi$ is also increasing, then (1.4)s a refinement of Jensen's inequality.

This paper is organized as follows. The main result (Theorem 1) is stated and proved in Sec. 2. In Sec. 3, we present, derive and discuss some consequences of the main result, by, in particular, giving new variants and generalizations of (1.1) and (1.2); see especially Theorem 2.

\section{A GENERAL VERSION OF HERMITE-HADAMARD INEQUALITY FOR $N$-QUASICONVEX FUNCTIONS}

Our main result in this section reads:

Theorem 1. Let $\varphi:[a, b] \rightarrow \mathbb{R}, a \geq 0$, be differentiable, convex and $\psi_{N}(x)=x^{N} \varphi(x), N=1,2, \ldots$. Let $p:[a, b] \rightarrow \mathbb{R}$ be nonnegative, integrable, and symmetric with respect to $x=(a+b) / 2$.

Then

$$
\begin{aligned}
\int_{a}^{b} \psi_{N}(x) p(x) d x \geq & \psi_{N}\left(\frac{a+b}{2}\right) \int_{a}^{b} p(x) d x+\int_{a}^{b}\left(x-\frac{a+b}{2}\right)^{2} \sum_{k=1}^{N} x^{k-1} \psi_{N-k}^{\prime}\left(\frac{a+b}{2}\right) p(x) d x \\
= & \psi_{N}\left(\frac{a+b}{2}\right) \int_{a}^{b} p(x) d x \\
& \quad+\int_{a}^{b}\left(x-\frac{a+b}{2}\right)^{2}\left(\left.\frac{\partial}{\partial \bar{x}}\left(\frac{x^{N}-\bar{x}^{N}}{x-\bar{x}} \varphi(\bar{x})\right)\right|_{\bar{x}=(a+b) / 2}\right) p(x) d x \\
\int_{a}^{b} \psi_{N}(x) p(x) d x \leq & \frac{\psi_{N}(a)+\psi_{N}(b)}{2} \int_{a}^{b} p(x) d x \\
& -\frac{1}{(b-a)} \sum_{k=1}^{N} \int_{a}^{b}(x-a)(b-x) \psi_{N-k}^{\prime}(x)\left((b-x) b^{k-1}+(x-a) a^{k-1}\right) p(x) d x
\end{aligned}
$$




$$
\begin{aligned}
& =\frac{\psi_{N}(a)+\psi_{N}(b)}{2} \int_{a}^{b} p(x) d x \\
& -\frac{1}{(b-a)} \int_{a}^{b}\left[(x-a)(b-x)^{2} \frac{\partial}{\partial x}\left(\frac{b^{N}-x^{N}}{b-x} \varphi(x)\right)\right. \\
& \left.\quad+(x-a)^{2}(b-x) \frac{\partial}{\partial x}\left(\frac{x^{N}-a^{N}}{x-a} \varphi(x)\right)\right] p(x) d x .
\end{aligned}
$$

Proof. From (1.3), for $a \leq x \leq b$, we obtain

$$
\begin{aligned}
& \psi_{N}(b)-\psi_{N}(x) \geq \psi_{N}^{\prime}(x)(b-x)+(b-x)^{2} \sum_{k=1}^{N} b^{k-1} \psi_{N-k}^{\prime}(x) \\
& \psi_{N}(a)-\psi_{N}(x) \geq \psi_{N}^{\prime}(x)(a-x)+(x-a)^{2} \sum_{k=1}^{N} a^{k-1} \psi_{N-k}^{\prime}(x) .
\end{aligned}
$$

Multiplying (2.3) by $(x-a) /(b-a)$ and (2.4)) by $(b-x) /(b-a)$, adding up, and using the relation $b(x-a) /(b-a)+a(b-x) /(b-a)=x$, we find that

$$
\begin{aligned}
& \frac{x-a}{b-a} \psi_{N}(b)+\frac{b-x}{b-a} \psi_{N}(a)-\psi_{N}(x) \\
& \quad \geq \frac{(x-a)(b-x)}{b-a} \sum_{k=1}^{N}\left((b-x) b^{k-1}+(x-a) a^{k-1}\right) \psi_{N-k}^{\prime}(x) .
\end{aligned}
$$

Now we add (2.3) multiplied by $(b-x) /(b-a)$ and (2.4) multiplied by $(x-a) /(b-a)$, obtaining

$$
\begin{aligned}
& \frac{b-x}{b-a} \psi_{N}(b)+\frac{x-a}{b-a} \psi_{N}(a)-\psi_{N}(a+b-x) \\
& \quad \geq \frac{(b-x)(x-a)}{b-a} \sum_{k=1}^{N}\left((x-a) b^{k-1}+(b-x) a^{k-1}\right) \psi_{N-k}^{\prime}(a+b-x)
\end{aligned}
$$

where $\psi_{N-k}^{\prime}(a+b-x)$ means $d \psi_{N-k}(z) / d z$ at $z=a+b-x$.

Adding (2.5) and (2.6), multiplying it by $p(x)$, and taking into consideration that $p(x)=p(a+b-x)$, we obtain

$$
\begin{aligned}
\left(\psi_{N}(b)+\right. & \left.\psi_{N}(a)\right) p(x) \\
\geq & \psi_{N}(x) p(x)+\psi_{N}(a+b-x) p(a+b-x) \\
& +\frac{(x-a)(b-x)}{b-a} \sum_{k=1}^{N}\left((b-x) b^{k-1}+(x-a) a^{k-1}\right) \psi_{N-k}^{\prime}(x) p(x) \\
& +\frac{(x-a)(b-x)}{b-a} \sum_{k=1}^{N}\left((x-a) b^{k-1}+(b-x) a^{k-1}\right) \psi_{N-k}^{\prime}(a+b-x) p(a+b-x) .
\end{aligned}
$$

Next, we make a change of variables $x \rightarrow a+b-x$ and integrate over the interval $[a,(a+b) / 2]$. After some calculations, we obtain

$$
\begin{gathered}
\int_{a}^{(a+b) / 2} \frac{(x-a)(b-x)}{b-a} \sum_{k=1}^{N}\left((x-a) b^{k-1}+(b-x) a^{k-1}\right) \psi_{N-k}^{\prime}(a+b-x) p(a+b-x) d x \\
=\int_{(a+b) / 2}^{b} \frac{(x-a)(b-x)}{b-a} \sum_{k=1}^{N}\left((b-x) b^{k-1}+(x-a) a^{k-1}\right) \psi_{N-k}^{\prime}(x) p(x) d x
\end{gathered}
$$




$$
\int_{a}^{(a+b) / 2}\left(\psi_{N}(b)+\psi_{N}(a)\right) p(x) d x=\frac{\left(\psi_{N}(b)+\psi_{N}(a)\right)}{2} \int_{a}^{b} p(x) d x .
$$

Now, by using (2.8) and (2.9), and by integrating (2.7) from $a$ to $(a+b) / 2$, we find

$$
\begin{aligned}
& \frac{\psi_{N}(b)+\psi_{N}(a)}{2} \int_{a}^{b} p(x) d x=\int_{a}^{(a+b) / 2}\left(\psi_{N}(b)+\psi_{N}(a)\right) p(x) d x \\
& \geq \int_{a}^{(a+b) / 2} \psi_{N}(x) p(x) d x \\
& \quad+\frac{1}{b-a} \int_{a}^{(a+b) / 2}(x-a)(b-x) \sum_{k=1}^{N}\left((b-x) b^{k-1}+(x-a) a^{k-1}\right) \psi_{N-k}^{\prime}(x) p(x) d x \\
& \quad+\int_{(a+b) / 2}^{b} \psi_{N}(x) p(x) d x \\
& \quad+\frac{1}{b-a} \int_{(a+b) / 2}^{b}(x-a)(b-x) \sum_{k=1}^{N}\left((b-x) b^{k-1}+(x-a) a^{k-1}\right) \psi_{N-k}^{\prime}(x) p(x) d x .
\end{aligned}
$$

This inequality and elementary calculations imply (2.2).

Now we prove the lower bound for $\int_{a}^{b} \psi_{N}(x) p(x) d x$, expressed in (2.1). To find this bound, we first use (1.3) with $x=(a+b) / 2$, obtaining

$$
\begin{aligned}
\psi_{N}(x) & -\psi_{N}\left(\frac{a+b}{2}\right) \\
& \geq \psi_{N}^{\prime}\left(\frac{a+b}{2}\right)\left(x-\frac{a+b}{2}\right)+\left(x-\frac{a+b}{2}\right)^{2} \sum_{k=1}^{N} x^{k-1} \psi_{N-k}^{\prime}\left(\frac{a+b}{2}\right) .
\end{aligned}
$$

Next, we replace $x$ with $a+b-x$ and find

$$
\begin{aligned}
& \psi_{N}(a+b-x)-\psi_{N}\left(\frac{a+b}{2}\right) \\
& \quad \geq \psi_{N}^{\prime}\left(\frac{a+b}{2}\right)\left(\frac{a+b}{2}-x\right)+\left(x-\frac{a+b}{2}\right)^{2} \sum_{k=1}^{N}(a+b-x)^{k-1} \psi_{N-k}^{\prime}\left(\frac{a+b}{2}\right) .
\end{aligned}
$$

Adding (2.10) and (2.11), we obtain

$$
\begin{aligned}
\psi_{N}(x)+\psi_{N}(a+b-x) \geq 2 & \psi_{N}\left(\frac{a+b}{2}\right)+\left(x-\frac{a+b}{2}\right)^{2} \sum_{k=1}^{N} x^{k-1} \psi_{N-k}^{\prime}\left(\frac{a+b}{2}\right) \\
& +\left(x-\frac{a+b}{2}\right)^{2} \sum_{k=1}^{N}(a+b-x)^{k-1} \psi_{N-k}^{\prime}\left(\frac{a+b}{2}\right) .
\end{aligned}
$$

Finally, multiplying (2.12) by the nonnegative and symmetric function $p(x)$ and integrating, we see that

$$
\begin{aligned}
& \int_{a}^{b} \psi_{N}(x) p(x) d x=\int_{a}^{(a+b) / 2} \psi_{N}(x) p(x) d x+\int_{(a+b) / 2}^{b} \psi_{N}(x) p(x) d x \\
& \quad=\int_{a}^{(a+b) / 2}\left(\psi_{N}(x)+\psi_{N}(a+b-x)\right) p(x) d x \\
& \quad \geq \int_{a}^{(a+b) / 2}\left(\psi_{N}\left(\frac{a+b}{2}\right)+\left(x-\frac{a+b}{2}\right)^{2} \sum_{k=1}^{N} x^{k-1} \psi_{N-k}^{\prime}\left(\frac{a+b}{2}\right)\right) p(x) d x
\end{aligned}
$$




$$
\begin{aligned}
& +\int_{a}^{(a+b) / 2}\left[\psi_{N}\left(\frac{a+b}{2}\right)\right. \\
& \left.\quad+\left(x-\frac{a+b}{2}\right)^{2} \sum_{k=1}^{N}(a+b-x)^{k-1} \psi_{N-k}^{\prime}\left(\frac{a+b}{2}\right)\right] p(a+b-x) d x \\
& =\int_{a}^{(a+b) / 2}\left(\psi_{N}\left(\frac{a+b}{2}\right)+\left(x-\frac{a+b}{2}\right)^{2} \sum_{k=1}^{N} x^{k-1} \psi_{N-k}^{\prime}\left(\frac{a+b}{2}\right)\right) p(x) d x \\
& +\int_{(a+b) / 2}^{b}\left(\psi_{N}\left(\frac{a+b}{2}\right)+\left(x-\frac{a+b}{2}\right)^{2} \sum_{k=1}^{N} x^{k-1} \psi_{N-k}^{\prime}\left(\frac{a+b}{2}\right)\right) p(x) d x \\
& =\int_{a}^{b}\left(\psi_{N}\left(\frac{a+b}{2}\right)+\left(x-\frac{a+b}{2}\right)^{2} \sum_{k=1}^{N} x^{k-1} \psi_{N-k}^{\prime}\left(\frac{a+b}{2}\right)\right) p(x) d x .
\end{aligned}
$$

This inequality obviously implies (2.1) and the proof is complete.

\section{SOME CONSEQUENCES OF THEOREM 1}

In this section, we present and derive a number of new variants and refinements of (1.1) and (1.2). First we point out some more or less obvious consequences of Theorem 1 for $N=1$.

Corollary 1. If $\varphi:[a, b] \rightarrow \mathbb{R}, a \geq 0$, is a differentiable and convex function and $\psi_{1}(x)=x \varphi(x)$, then

$$
\begin{gathered}
\psi_{1}\left(\frac{a+b}{2}\right) \int_{a}^{b} p(x) d x+\varphi^{\prime}\left(\frac{a+b}{2}\right) \int_{a}^{b}\left(x-\frac{a+b}{2}\right)^{2} p(x) d x \leq \int_{a}^{b} \psi_{1}(x) p(x) d x \\
\leq \frac{\psi_{1}(a)+\psi_{1}(b)}{2} \int_{a}^{b} p(x) d x-\int_{a}^{b} \varphi^{\prime}(x)(b-x)(x-a) p(x) d x,
\end{gathered}
$$

where $p:[a, b] \rightarrow \mathbb{R}$ is nonnegative, integrable, and symmetric with respect to $x=(a+b) / 2$.

Note that for $p(x)=1,(3.1)$ reads:

Example 1. Let $\varphi$ and $\psi_{1}$ be defined as in Corollary 1. Then

$$
\begin{aligned}
& \psi_{1}\left(\frac{a+b}{2}\right)+\frac{1}{12} \varphi^{\prime}\left(\frac{a+b}{2}\right)(b-a)^{2} \\
& \quad \leq \frac{1}{b-a} \int_{a}^{b} \psi_{1}(x) d x \leq \frac{\psi_{1}(a)+\psi_{1}(b)}{2}-\frac{1}{b-a} \int_{a}^{b} \varphi^{\prime}(x)(b-x)(x-a) d x .
\end{aligned}
$$

Remark 1. When $\varphi$ is a convex and increasing function and, therefore, $\psi_{1}$ is also convex, we have

(a) inequalities (3.1) are refinements of the Fejér inequality (1.2));

(b) inequalities (3.2) are refinements of the Hermite-Hadamard inequality(1.1).

For $N=1$, when both $\varphi$ and $\varphi^{\prime}$ are convex functions, we can also present the following.

Example 2. Let $\varphi:[a, b] \rightarrow \mathbb{R}, a \geq 0$, be differentiable, convex, and increasing, and let $\psi_{1}(x)=x \varphi(x)$. Let $\varphi^{\prime}$ be also convex. Then

$$
\begin{aligned}
& \psi_{1}\left(\frac{a+b}{2}\right)+\frac{1}{12} \varphi^{\prime}\left(\frac{a+b}{2}\right)(b-a)^{2} \\
& \quad \leq \frac{1}{b-a} \int_{a}^{b} \psi_{1}(x) d x \leq \frac{\psi_{1}(a)+\psi_{1}(b)}{2}-\frac{1}{6} \varphi^{\prime}\left(\frac{a+b}{2}\right)(b-a)^{2} .
\end{aligned}
$$


In fact, because $\varphi^{\prime}$ is convex and as $(b-x)(x-a)$ is nonnegative, symmetric in the interval $[a, b]$, it follows from the Fejér inequality (1.2) for the convex function $\varphi^{\prime}$ that

$$
\begin{aligned}
\varphi^{\prime}\left(\frac{a+b}{2}\right) \frac{(b-a)^{2}}{6} & =\varphi^{\prime}\left(\frac{a+b}{2}\right) \int_{a}^{b} \frac{(b-x)(x-a)}{b-a} d x \\
& \leq \frac{1}{b-a} \int_{a}^{b} \varphi^{\prime}(x)(b-x)(x-a) d x
\end{aligned}
$$

By using the estimate (3.4) and the right-hand side of inequality (3.2), we obtain the right-hand side inequality in (3.3). The left-hand inequality in (3.3) is the same as that in (3.2).

Remark 2. If $\varphi$ is convex and $\varphi^{\prime}$ is concave, we obtain, in the same way as in Example 2, the inequalities

$$
\begin{aligned}
& \psi_{1}\left(\frac{a+b}{2}\right)+\frac{1}{12} \varphi^{\prime}\left(\frac{a+b}{2}\right)(b-a)^{2} \\
& \quad \leq \frac{1}{b-a} \int_{a}^{b} \psi_{1}(x) d x \leq \frac{\psi_{1}(a)+\psi_{1}(b)}{2}-\frac{1}{6} \frac{\varphi^{\prime}(a)+\varphi^{\prime}(b)}{2}(b-a)^{2} .
\end{aligned}
$$

Next, we observe that under the condition that $p(x)$ is integrable, nonnegative, and symmetric on $[a, b]$, the same is true for $(b-x)(x-a) p(x)$, which leads to the following example.

Example 3. Let $\varphi:[a, b] \rightarrow \mathbb{R}, a \geq 0$, be differentiable, convex, and increasing, and let $\psi_{1}(x)=x \varphi(x)$. Let $\varphi^{\prime}$ be convex and $p(x)$ be integrable, nonnegative and symmetric on $[a, b]$. Then

$$
\begin{gathered}
\psi_{1}\left(\frac{a+b}{2}\right) \int_{a}^{b} p(x) d x+\varphi^{\prime}\left(\frac{a+b}{2}\right) \int_{a}^{b}\left(x-\frac{a+b}{2}\right)^{2} p(x) d x \leq \int_{a}^{b} \psi_{1}(x) p(x) d x \\
\leq \frac{\psi_{1}(a)+\psi_{1}(b)}{2} \int_{a}^{b} p(x) d x-\varphi^{\prime}\left(\frac{a+b}{2}\right) \int_{a}^{b}(b-x)(x-a) p(x) d x .
\end{gathered}
$$

We now state the following less obvious refinement of the Hermite-Hadamard inequality (1.1).

Corollary 2. Let $\psi_{1}$ be a 1-quasiconvex function, i.e., $\psi_{1}(x)=x \varphi(x)$, where $\varphi$ is differentiable and convex on $[a, b], a \geq 0$. Then

$$
\begin{aligned}
& \psi_{1}\left(\frac{a+b}{2}\right)+\frac{1}{12} \varphi^{\prime}\left(\frac{a+b}{2}\right)(b-a)^{2} \leq \frac{1}{b-a} \int_{a}^{b} \psi_{1}(x) d x \\
& \quad \leq \frac{\psi_{1}(a)+\psi_{1}(b)}{6}+\frac{a+b}{3} \frac{1}{b-a} \int_{a}^{b} \varphi(x) d x \leq \frac{\psi_{1}(a)+\psi_{1}(b)}{2}-\frac{(b-a)(\varphi(b)-\varphi(a))}{6} .
\end{aligned}
$$

Moreover,

$$
\begin{aligned}
& \frac{1}{b-a} \int_{a}^{b} \psi_{1}(x) d x-\frac{\psi_{1}(a)+\psi_{1}(b)}{2}+\frac{1}{b-a} \int_{a}^{b} \varphi^{\prime}(x)(b-x)(x-a) d x \\
& \quad=3\left[\frac{1}{b-a} \int_{a}^{b} \psi_{1}(x) d x-\frac{\psi_{1}(a)+\psi_{1}(b)}{6}-\frac{(a+b)}{3} \frac{1}{b-a} \int_{a}^{b} \varphi(x) d x\right] \leq 0 .
\end{aligned}
$$

Proof. Since

$$
\begin{aligned}
\int_{a}^{b} \varphi^{\prime}(x)(b-x)(x-a) d x & =-\int_{a}^{b} \varphi(x)(a+b-2 x) d x \\
& =2 \int_{a}^{b} \psi_{1}(x) d x-(a+b) \int_{a}^{b} \varphi(x) d x
\end{aligned}
$$

we can rewrite the right-hand side inequality in (3.2) as

$$
\frac{1}{b-a} \int_{a}^{b} \psi_{1}(x) d x \leq \frac{\psi_{1}(a)+\psi_{1}(b)}{2}-2 \frac{1}{b-a} \int_{a}^{b} \psi_{1}(x) d x+(a+b) \frac{1}{b-a} \int_{a}^{b} \varphi(x) d x
$$


and, from this inequality, by rearranging its terms, we obtain

$$
\frac{1}{b-a} \int_{a}^{b} \psi_{1}(x) d x \leq \frac{\psi_{1}(a)+\psi_{1}(b)}{6}+\frac{(a+b)}{3} \frac{1}{b-a} \int_{a}^{b} \varphi(x) d x .
$$

Moreover, from the convexity of $\varphi$, by using the Hermite-Hadamard inequality $(1.1)$ and the fact that $\psi_{1}(x)=x \varphi(x)$, we see that

$$
\frac{\psi_{1}(a)+\psi_{1}(b)}{6}+\frac{(a+b)}{3} \frac{1}{b-a} \int_{a}^{b} \varphi(x) d x \leq \frac{\psi_{1}(a)+\psi_{1}(b)}{2}-\frac{(b-a)(\varphi(b)-\varphi(a))}{6} .
$$

By combining (3.8) and (3.9) and using the left-hand side inequality of (3.2), we get (3.5).

Next, we again use (3.7) to conclude that

$$
\begin{gathered}
\frac{1}{b-a} \int_{a}^{b} \psi_{1}(x) d x-\frac{\psi_{1}(a)+\psi_{1}(b)}{2}+\frac{1}{b-a} \int_{a}^{b} \varphi^{\prime}(x)(b-x)(x-a) d x \\
-\left[\frac{1}{b-a} \int_{a}^{b} \psi_{1}(x) d x-\frac{\psi_{1}(a)+\psi_{1}(b)}{6}-\frac{(a+b)}{3} \frac{1}{b-a} \int_{a}^{b} \varphi(x) d x\right] \\
=\left[\frac{\psi_{1}(a)+\psi_{1}(b)}{6}+\frac{(a+b)}{3} \frac{1}{b-a} \int_{a}^{b} \varphi(x) d x\right] \\
\quad-\frac{\psi_{1}(a)+\psi_{1}(b)}{2}+\frac{1}{b-a} \int_{a}^{b} \varphi^{\prime}(x)(b-x)(x-a) d x
\end{gathered}
$$

Finally, we use (3.5) and make a simple calculation to obtain (3.6); the proof is complete.

Remark 3. Note that (3.5) yields a refinement of the right-hand side of the Hermite-Hadamard inequality (1.1) (it suffices that $\varphi$ be convex on $[a, b]$ and $\varphi(b) \geq \varphi(a)$ ). For the refinement of the left-hand side of the Hermite-Hadamard inequality (1.1), it suffices that $\varphi$ be convex on $[a, b]$ and $\varphi^{\prime}((a+b) / 2) \geq 0$.

Example 4. If the conditions in Remark 3 are satisfied, then the inequalities

$$
\begin{aligned}
\psi_{1}\left(\frac{a+b}{2}\right) & \leq \psi_{1}\left(\frac{a+b}{2}\right)+\frac{1}{12} \varphi^{\prime}\left(\frac{a+b}{2}\right)(b-a)^{2} \leq \frac{1}{b-a} \int_{a}^{b} \psi_{1}(x) d x \\
& \leq \frac{\psi_{1}(a)+\psi_{1}(b)}{6}+\frac{a+b}{3} \frac{1}{b-a} \int_{a}^{b} \varphi(x) d x \\
& \leq \frac{\psi_{1}(a)+\psi_{1}(b)}{2}-\frac{(b-a)(\varphi(b)-\varphi(a))}{6} \leq \frac{\psi_{1}(a)+\psi_{1}(b)}{2}
\end{aligned}
$$

hold, which are refinements of the Hermite-Hadamard inequality (1.1).

We continue this section by giving some similar results also for values of $N$ other than $N=1$.

Example 5. For $N=2,3, \ldots$, inequalities (2.1) and (2.2) are refinements of the Fejér inequality (1.2). If $\varphi$ is a convex, increasing and nonnegative function and if also $p(x) \equiv 1$, then it follows that

$$
\begin{aligned}
& \psi_{N}\left(\frac{a+b}{2}\right)+\frac{1}{b-a} \int_{a}^{b}\left(x-\frac{a+b}{2}\right)^{2}\left(\left.\frac{\partial}{\partial \bar{x}}\left(\frac{x^{N}-\bar{x}^{N}}{x-\bar{x}} \varphi(\bar{x})\right)\right|_{\bar{x}=(a+b) / 2}\right) d x \\
& \quad=\psi_{N}\left(\frac{a+b}{2}\right)+\frac{1}{b-a} \int_{a}^{b}\left(x-\frac{a+b}{2}\right)^{2} \sum_{k=1}^{N} x^{k-1} \psi_{N-k}^{\prime}\left(\frac{a+b}{2}\right) d x \\
& \quad \leq \frac{1}{b-a} \int_{a}^{b} \psi_{N}(x) d x \\
& \quad \leq \frac{\psi_{N}(a)+\psi_{N}(b)}{2}-\sum_{k=1}^{N} \int_{a}^{b} \frac{(x-a)(b-x)\left((b-x) b^{k-1}+(x-a) a^{k-1}\right)}{(b-a)^{2}} \psi_{N-k}^{\prime}(x) d x .
\end{aligned}
$$




$$
\begin{aligned}
=\frac{\psi_{N}(a)+\psi_{N}(b)}{2}-\frac{1}{(b-a)^{2}} \int_{a}^{b}\left[(x-a)(b-x)^{2} \frac{\partial}{\partial x}\left(\frac{b^{N}-x^{N}}{b-x} \varphi(x)\right)\right. \\
\left.+(x-a)^{2}(b-x) \frac{\partial}{\partial x}\left(\frac{x^{N}-a^{N}}{x-a} \varphi(x)\right)\right] d x
\end{aligned}
$$

which are refinements of the Hermite-Hadamard inequality (1.1), when $\varphi$ is a differentiable, nonnegative, nondecreasing, and convex function.

Next, we present the main result of this section, namely, a nondecreasing sequence of upper bounds of $(1 /(b-a)) \int_{a}^{b} \psi_{1}(x) d x$ by using the upper bounds of $(1 /(b-a)) \int_{a}^{b} \psi_{N}(x) d x$ from Example 5 .

Theorem 2. Let $\varphi:[a, b] \rightarrow \mathbb{R}, a \geq 0$, be a differentiable, convex function and $\psi_{N}(x)=x^{N} \varphi(x)$, $N=1,2,3, \ldots$, Then

$$
\begin{aligned}
& \frac{1}{b-a} \int_{a}^{b} \psi_{1}(x) d x \\
& \quad \leq \frac{(b-a)\left(\psi_{N}(a)+\psi_{N}(b)\right)}{6\left(b^{N}-a^{N}\right)}+\frac{\left(b^{N+1}-a^{N+1}\right)+2 a b\left(b^{N-1}-a^{N-1}\right)}{3\left(b^{N}-a^{N}\right)} \frac{1}{b-a} \int_{a}^{b} \varphi(x) d x:=H_{N} .
\end{aligned}
$$

Moreover, $\left\{H_{N}\right\}_{1}^{\infty}$ is a nonincreasing sequence of $N$. Especially, this yields that

$$
\begin{aligned}
& \frac{1}{b-a} \int_{a}^{b} \psi_{1}(x) d x \leq \frac{b-a}{6} \varphi(b)+\frac{b+2 a}{3} \frac{1}{b-a} \int_{a}^{b} \varphi(x) d x \\
& \quad \leq \frac{(b-a)\left(\psi_{N}(a)+\psi_{N}(b)\right)}{6\left(b^{N}-a^{N}\right)}+\frac{\left(b^{N+1}-a^{N+1}\right)+2 a b\left(b^{N-1}-a^{N-1}\right)}{3\left(b^{N}-a^{N}\right)} \frac{1}{b-a} \int_{a}^{b} \varphi(x) d x \\
& \quad \leq \frac{\psi_{1}(a)+\psi_{1}(b)}{6}+\frac{(b+a)}{3} \frac{1}{b-a} \int_{a}^{b} \varphi(x) d x \\
& \quad \leq \frac{\psi_{1}(a)+\psi_{1}(b)}{2}-\frac{(b-a)(\varphi(b)-\varphi(a))}{6} .
\end{aligned}
$$

Example 6. For $N=1$ (see Corollary 2 ) and for $N=2$, we have

$$
\begin{aligned}
\frac{1}{b-a} \int_{a}^{b} \psi_{1}(x) d x & \leq \frac{\psi_{2}(a)+\psi_{2}(b)}{6(a+b)}+\frac{(a+b)^{2}+a b}{3(a+b)} \frac{1}{a-b} \int_{a}^{b} \varphi(x) d x \\
& \leq \frac{\psi_{1}(a)+\psi_{1}(b)}{6}+\frac{a+b}{3} \frac{1}{b-a} \int_{a}^{b} \varphi(x) d x \\
& \leq \frac{\psi_{1}(a)+\psi_{1}(b)}{2}-\frac{(b-a)(\varphi(b)-\varphi(a))}{6} .
\end{aligned}
$$

Proof of Theorem 2. By using (2.2) with $p(x)=1$ and integrating by parts, we find that

$$
\begin{aligned}
& \frac{1}{b-a} \int_{a}^{b} \psi_{N}(x) d x \leq \frac{\psi_{N}(a)+\psi_{N}(b)}{2} \\
& \quad+\frac{1}{(b-a)^{2}} \sum_{k=1}^{N} \int_{a}^{b} \psi_{N-k}(x)\left((x-a)(b-x)\left((b-x) b^{k-1}+(x-a) a^{k-1}\right)\right)^{\prime} d x .
\end{aligned}
$$

Denote

$$
T_{k}=\int_{a}^{b} \psi_{N-k}(x)\left((x-a)(b-x)\left((b-x) b^{k-1}+(x-a) a^{k-1}\right)\right)^{\prime} d x, \quad k=1,2, \ldots, N .
$$


After some elementary computations, we obtain

$$
\begin{gathered}
\sum_{k=1}^{N} T_{k}=\sum_{k=1}^{N}\left[\left(\left(b^{k}-a^{k}\right)(a+b)+a b\left(b^{k-1}-a^{k-1}\right)\right) \int_{a}^{b} \psi_{N-k}(x) d x\right. \\
-2\left(b^{k}-a^{k}+(a+b)\left(b^{k-1}-a^{k-1}\right)\right) \int_{a}^{b} \psi_{N+1-k}(x) d x \\
\left.+3\left(b^{k-1}-a^{k-1}\right) \int_{a}^{b} \psi_{N+2-k}(x) d x\right]
\end{gathered}
$$

We can rewrite $(3.15)$ as

$$
\begin{aligned}
\sum_{k=1}^{N} T_{k}= & \sum_{k=1}^{N}\left(\left(b^{k}-a^{k}\right)(a+b)+a b\left(b^{k-1}-a^{k-1}\right)\right) \int_{a}^{b} \psi_{N-k}(x) d x \\
& -\sum_{k=0}^{N-1} 2\left(b^{k+1}-a^{k+1}+(a+b)\left(b^{k}-a^{k}\right)\right) \int_{a}^{b} \psi_{N-k}(x) d x \\
& +\sum_{k=-1}^{N-2} 3\left(b^{k+1}-a^{k+1}\right) \int_{a}^{b} \psi_{N-k}(x) d x
\end{aligned}
$$

which is the same as

$$
\begin{aligned}
\sum_{k=1}^{N} T_{k}=\sum_{k=1}^{N-2}\left[\left(\left(b^{k}-a^{k}\right)(a+b)+a b\left(b^{k-1}-a^{k-1}\right)\right)-2\left(b^{k+1}-a^{k+1}+(a+b)\left(b^{k}-a^{k}\right)\right)\right. \\
\left.\quad+3\left(b^{k+1}-a^{k+1}\right)\right] \int_{a}^{b} \psi_{N-k}(x) d x \\
\quad+\left(\left(b^{N-1}-a^{N-1}\right)(a+b)+a b\left(b^{N-2}-a^{N-2}\right)\right) \int_{a}^{b} \psi_{1}(x) d x \\
\quad+\left(\left(b^{N}-a^{N}\right)(a+b)+a b\left(b^{N-1}-a^{N-1}\right)\right) \int_{a}^{b} \psi_{0}(x) d x \\
\quad-2\left(b^{N}-a^{N}+(a+b)\left(b^{N-1}-a^{N-1}\right)\right) \int_{a}^{b} \psi_{1}(x) d x-2\left(b^{1}-a^{1}\right) \int_{a}^{b} \psi_{N}(x) d x \\
\quad+3\left(b^{1}-a^{1}\right) \int_{a}^{b} \psi_{N}(x) d x+3\left(b^{0}-a^{0}\right) \int_{a}^{b} \psi_{N+1}(x) d x
\end{aligned}
$$

Next, we add the terms that include $\int_{a}^{b} \psi_{N-j}(x) d x, j=1, \ldots, N$, in (3.16)) and see that for $j=1, \ldots, N-2$, the coefficients of $\int_{a}^{b} \psi_{N-j}(x) d x$ sum up to zero because

$$
\begin{aligned}
& {\left[\left(\left(b^{j}-a^{j}\right)(a+b)+a b\left(b^{j-1}-a^{j-1}\right)\right)\right.} \\
& \left.\quad-2\left(b^{j+1}-a^{j+1}+(a+b)\left(b^{j+1-1}-a^{j+1-1}\right)\right)+3\left(b^{j+2-1}-a^{j+2-1}\right)\right]=0 .
\end{aligned}
$$

Now we add the coefficients of $\int_{a}^{b} \psi_{N}(x) d x$ and $\int_{a}^{b} \psi_{N+1}(x) d x$ obtained from $T_{1}$ and $T_{2}$. The coefficient of $\int_{a}^{b} \psi_{N}(x) d x$ in $T_{1}$ is $(-2)(b-a)$, and the coefficient of $\int_{a}^{b} \psi_{N}(x) d x$ in $T_{2}$ is $3(b-a)$, together the coefficient of $\int_{a}^{b} \psi_{N}(x) d x$ is $(b-a)$.

Also, from $T_{1}$ we see that the coefficient of $\int_{a}^{b} \psi_{N+1}(x) d x$ is zero.

Now note that the contribution of $T_{N-1}$ and $T_{N}$ to $\int_{a}^{b} \psi_{N-j}(x) d x$ for $j=N-1$ and $j=N$ is the contribution to the coefficient of $\int_{a}^{b} \psi_{1}(x) d x$, which is equal to

$$
\begin{aligned}
& \left(\left(b^{N-1}-a^{N-1}\right)(a+b)+a b\left(b^{N-1-1}-a^{N-1-1}\right)\right)-2\left(b^{N}-a^{N}+(a+b)\left(b^{N-1}-a^{N-1}\right)\right) \\
& \quad=-3\left(b^{N}-a^{N}\right),
\end{aligned}
$$


and the coefficients of

$$
\int_{a}^{b} \psi_{0}(x) d x=\int_{a}^{b} \varphi(x) d x
$$

appears only in $T_{N}$ and is equal to

$$
(a+b)\left(b^{N}-a^{N}\right)+a b\left(b^{N-1}-a^{N-1}\right)=2 a b\left(b^{N-1}-a^{N-1}\right)+b^{N+1}-a^{N+1} .
$$

Therefore we get that

$$
\begin{aligned}
\sum_{k=1}^{N} T_{k}= & 0 \int_{a}^{b} \psi_{N+1}(x) d x+(b-a) \int_{a}^{b} \psi_{N}(x) d x+\sum_{k=1}^{N-2} 0 \int_{a}^{b} \psi_{N-k}(x) d x \\
& -3\left(b^{N}-a^{N}\right) \int_{a}^{b} \psi_{1}(x) d x+\left(\left(b^{N+1}-a^{N+1}\right)+2 a b\left(b^{N-1}-a^{N-1}\right)\right) \int_{a}^{b} \varphi(x) d x .
\end{aligned}
$$

By combining (3.14) with (3.17), and making a simple computation, we obtain (3.11).

To prove (3.12), we use the convexity of $\varphi$ and make some elementary calculations to derive that

$$
\begin{aligned}
& \frac{(b-a)\left(\psi_{N}(a)+\psi_{N}(b)\right)}{6\left(b^{N}-a^{N}\right)}+\frac{\left(b^{N+1}-a^{N+1}\right)+2 a b\left(b^{N-1}-a^{N-1}\right)}{3\left(b^{N}-a^{N}\right)} \frac{1}{b-a} \int_{a}^{b} \varphi(x) d x \\
& -\left[\frac{(b-a)\left(\psi_{N-1}(a)+\psi_{N-1}(b)\right)}{6\left(b^{N-1}-a^{N-1}\right)}+\frac{\left(b^{N}-a^{N}\right)+2 a b\left(b^{N-2}-a^{N-2}\right)}{3\left(b^{N-1}-a^{N-1}\right)} \frac{1}{b-a} \int_{a}^{b} \varphi(x) d x\right] \\
& =\frac{a^{N-1} b^{N-1}(b-a)^{2}}{3\left(b^{N}-a^{N}\right)\left(\left(b^{N-1}-a^{N-1}\right)\right)}\left(\frac{1}{b-a} \int_{a}^{b} \varphi(x) d x-\frac{\varphi(a)+\varphi(b)}{2}\right) \leq 0 .
\end{aligned}
$$

The last inequality in (3.18) is just (1.1). Therefore from (3.18) it follows that the sequence $\left\{H_{N}\right\}_{1}^{\infty}$ is nonincreasing. The second inequality in (3.12) is just (3.11). The third inequality in (3.12) follows from the fact that $\left\{H_{N}\right\}_{1}^{\infty}$ is nonincreasing. For the fourth inequality (see Corollary 2), we again use the monotonicity of $\left\{H_{N}\right\}_{1}^{\infty}$. From the fact that this sequence is bounded below and by letting $N \rightarrow \infty$, we conclude that the first inequality in (3.12) also holds. The proof is complete.

Finally, we state the following monotonicity and comparison result.

Proposition. Let $\varphi$ be a differentiable, nonnegative, increasing, and convex function. Then, when we consider the $N$-quasiconvex function $\psi_{N}=\varphi(x) x^{N}$, the lower bound in (3.10) of $(1 /(b-a)) \int_{a}^{b} \psi_{N}(x) d x$ is greater than the lower bound for the $(N-J)$-quasiconvex function $(1 /(b-a)) \int_{a}^{b} \phi_{0, J}(x) x^{N-J} d x$ derived from (3.10), where $\phi_{0, J}(x)=\varphi(x) x^{J}$ and the bound for $(1 /(b-a)) \int_{a}^{b} \phi_{0, J}(x) x^{N-J} d x$ is nonincreasing with $J, 0 \leq J \leq N$.

Similarly, the upper bound for $(1 /(b-a)) \int_{a}^{b} \phi_{0, J}(x) x^{N-J} d x$ is increasing with $J, 0 \leq J \leq N$.

Proof. By using (3.10) for $\phi_{0, J}(x) x^{N-J}$, which like $\varphi$ is also a differentiable, convex, and increasing function, we see that the lower bound is

$$
\begin{aligned}
& \frac{1}{b-a} \int_{a}^{b} \psi_{N}(x) d x=\frac{1}{b-a} \int_{a}^{b} \phi_{0, J}(x) x^{N-J} d x \\
& \geq \phi_{0, J}\left(\frac{a+b}{2}\right)\left(\frac{a+b}{2}\right)^{N-J} \\
& \quad+\frac{1}{b-a} \int_{a}^{b}\left(x-\frac{a+b}{2}\right)^{2} \sum_{k=1}^{N-J} x^{k-1} \phi_{0, N-J-k}^{\prime}\left(\frac{a+b}{2}\right) d x \\
& =\psi_{N}\left(\frac{a+b}{2}\right)+\frac{1}{b-a} \int_{a}^{b}\left(x-\frac{a+b}{2}\right)^{2} \sum_{k=1}^{N-J} x^{k-1} \psi_{N-k}^{\prime}\left(\frac{a+b}{2}\right) d x
\end{aligned}
$$


The last equality in (3.19) holds because

$$
\phi_{0, N-J-k}(x)=\left(\varphi(x) x^{J}\right) x^{N-J-k}=\varphi(x) x^{N-k}=\psi_{N-k}(x) .
$$

Therefore, since it is clear that

$$
\frac{1}{b-a} \int_{a}^{b}\left(x-\frac{a+b}{2}\right)^{2} \sum_{k=1}^{N-J} x^{k-1} \phi_{0, N-k}^{\prime}\left(\frac{a+b}{2}\right) d x
$$

is decreasing with $J, 0 \leq J \leq N$, our claim about the monotonicity of the lower bound for

$$
\frac{1}{b-a} \int_{a}^{b} \psi_{N}(x) d x=\frac{1}{b-a} \int_{a}^{b} \phi_{0, J}(x) x^{N-J} d x
$$

is proved. Similarly, it can be proved that the upper bound for

$$
\frac{1}{b-a} \int_{a}^{b} \psi_{N}(x) d x=\frac{1}{b-a} \int_{a}^{b} \phi_{0, J}(x) x^{N-J} d x
$$

is increasing with $J$. The proof is complete.

\section{REFERENCES}

1. S. S. Dragomir, J. Pečarić, and L. E. Persson, "Some inequalities of Hadamard type," Soochow J. Math. 21 (3), 335-341 (1995).

2. S. S. Dragomir, " $n$-points inequalities of Hermite-Hadamard type for $h$-convex functions on linear spaces," Armen. J. Math. 8(1), 38-57 (2016).

3. S. Varošanec, "On $h$-convexity,” J. Math. Anal Appl. 326 (1), 303-311 (2007).

4. C. P. Niculescu and L. E. Persson, "Old and new on the Hermite-Hadamard inequality," Real Anal. Exchange 29 (2), 663-685 (2004).

5. C. P. Niculescu and L. E. Persson, Convex Functions and Their Applications. A Contemporary Approach, in CMS Books Math. (Springer, New York, 2006), Vol. 23.

6. S. Abramovich, "Hölder, Jensen, Minkowski, Jensen-Steffensen, and Slater-Pečarić inequalities derived through $N$-quasiconvexity," Math. Inequal. Appl. 19 (4), 1203-1226 (2016).

7. S. Abramovich and L. E. Persson, "Some new estimates of the 'Jensen gap'," J. Inequal. Appl. 2016 (39) (2016). 